\title{
Singularity-Invariant Leg Substitutions in Pentapods
}

\author{
Júlia Borràs and Federico Thomas
}

\begin{abstract}
A pentapod is usually defined as a 5-degree-offreedom fully-parallel manipulator with an axial spindle as moving platform. This kind of manipulators have revealed as an interesting alternative to serial robots handling axisymmetric tools. Their particular geometry permits that, in one tool axis, inclination angles of up to 90 degrees are possible thus overcoming the orientation limits of the classical Stewart platform.

This paper presents a solution to the problem of finding those changes in the location of the leg attachments of a pentapod that leave its singularity locus invariant. Although the solution to this problem does not provide a fully characterization of the singularities, it provides a lot of insight into its nature. It is shown, for example, that there are four different architectures for a pentapod with a completely different behavior from the point of view of their singularities.

The kinematics of pentaponds with coplanar attachments at the fixed base has previously been studied as rigid subassemblies of a Stewart platforms. In this paper, we treat the general case in which the base attachments are arbitrarily located in 3D space.
\end{abstract}

Index Terms-Pentapod, fully-parallel robots, singularities, singularity-invariant transformations.

\section{INTRODUCTION}

The Stewart platform consists of a base and a moving platform connected by six UPS (Universal-Prismatic-Spherical) legs, where the underline indicates that the prismatic joint is actuated. Thus, it is usually referenced to as a 6-UPS, or equivalently as a 6-SPU, parallel mechanism [7]. If one of these legs is eliminated to obtain a 5-DoF parallel robot, the resulting platform is clearly uncontrollable. For example, if the universal joints are properly aligned, the moving platform can freely rotate around the axis defined by these five aligned universal joints. Nevertheless, observe that in this particular case the uncontrolled motion is irrelevant in some applications. Indeed, there are important industrial tasks requiring a tool to be perpendicular to a $3 \mathrm{D}$ free-from surface along a given trajectory without caring about its axial orientation. They include, for example, 5-axis milling, laser-engraving, spray-based painting, and water-jet cutting. In this context, the study of the kinematics properties of 5SPU parallel robots with collinear universal joints becomes highly relevant for many applications [9]. Kong and Gosselin refer to this particular arrangement of five legs as a linebody component as it can always be considered as a rigid subassembly in a standard Stewart platform [4]. We will refer to it simply as a pentapod.

Júlia Borràs and Federico Thomas are with the Institut de Robòtica Informàtica Industrial, CSIC-UPC, Llorens Artigas 4-6, 08028 Barcelona, Spain, E-mails: \{jborras, fthomas\}@iri.upc.edu. This work has been partially supported by the Spanish Ministry of Education and Innovation, under the I+D project DPI2007-60858.

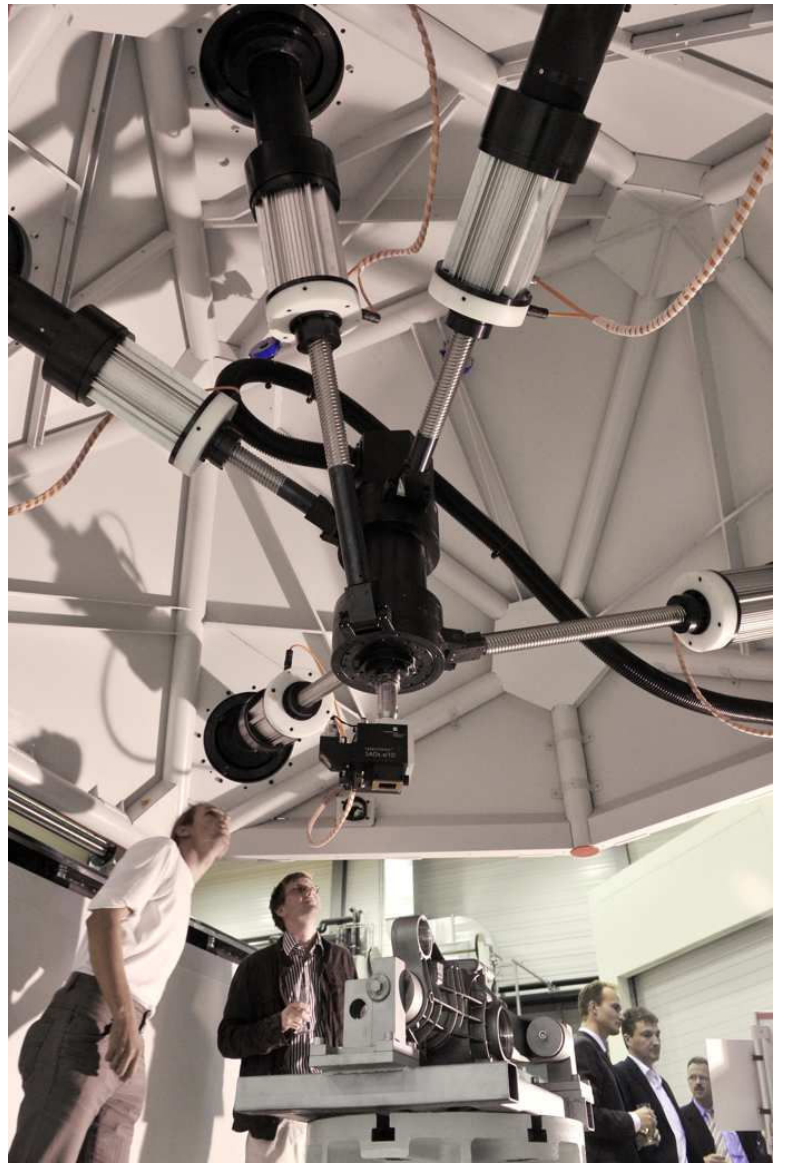

Fig. 1. A 5-axis milling machine, based on a pentapod, developed by Metrom Mechatronische Maschinen $\mathrm{GmbH}$ (reproduced with permission).

There are some variations on the basic described pentapod that consists in substituting the universal joints by two consecutive revolute joints. The axes of the last revolute joints remain collinear with the axis of the tool while the axis of the other revolute joint axis no longer intersect with the tool axis. This is the joint arrangement used by Metrom in its Pentapod machine (Fig. 1). This arrangement simplifies the construction of the resulting pentapod but its kinematic analysis is far from trivial. Actually, the solutions to its direct kinematics are given by the roots of a system of 5 polynomials of degree 4 together with a quadratic normalizing condition. Therefore, the number of solutions is not greater than 2048 [1], [10]. When this number is compared to the 16 possible direct kinematic solutions of the basic pentapod, one also gets an idea of the relative complexity between the singularity loci of the basic and the 
modified design.

In this paper, we concentrate ourselves in the analysis of the singularities of the basic design which, despite its practical interest, has received little attention in the past. Indeed, most of the related previous works deal with the case in which the spherical joints are coplanar. For example, Zhang and Song solved the forward kinematics problem for the coplanar case showing that it can have up to 8 assembly modes [11]. Husty and Karger studied the conditions for a pentapod with coplanar spherical joints to be architecturally singular [3]. More recently, Borràs and Thomas analyzed the role of cross-ratios between the location of the leg attachments in the characterization of architectural singularities, and in singularity-invariant architectural changes, also for the coplanar case [2].

The characterization of the singularities of spatial parallel mechanisms is, in general, a difficult task. To obtain this characterization, we will follow an indirect approach: we will find those changes in the location of the leg attachments of a pentapod that leave its singularity locus invariant.

Even when there is no known solution to a given mathematical problem, it is always possible to try to find the set of transformations to the problem that leave its solution invariant. Although this does not solve the problem itself, it provides a lot of insight into its nature. This way of thinking is at the root of the development of Group Theory and it is the one applied herein for the characterization of the singularity loci of pentapods.

The paper is organized as follows. The next section presents a new formulation for the characterization of the singularities of a pentapod. Section III presents a solution to the problem of changing the location of the attachments of one of the legs of the pentapod without altering its singularity locus. Section IV shows that there are four different architectures for a pentapod attending to the characteristics of the possible locations for the base attachment of the new leg in these singularity-invariant leg substitutions. Section V presents three examples corresponding to three different architectures. Finally, Section VII summarizes the main contributions.

\section{The Singularities of A Pentapod}

Let us consider a Stewart platform containing a pentapod, that is, a Stewart platform with five collinear attachments on its moving platform. Let $\Lambda$ denote the line to which these collinear attachments are incident. According to Fig. 2, the pose of $\Lambda$ is defined by a position vector of a point in it, $\mathbf{p}=\left(p_{x}, p_{y}, p_{z}\right)$, and a unit vector, $\mathbf{i}=(u, v, w)$, pointing in its direction. Finally, let leg $i$ have base and platform attachments with coordinates, in the base reference frame, $\mathbf{a}_{i}=\left(x_{i}, y_{i}, z_{i}\right)^{T}$ and $\mathbf{b}_{i}=\mathbf{p}+r_{i} \mathbf{i}, i=1, \ldots, 5$, respectively. In what follows, the base reference frame is chosen, without loss of generality, such that $x_{1}=y_{1}=$ $z_{1}=r_{1}=0$.

It is well-known that the singularities of a Stewart platform are given by the determinant of the Jacobian matrix whose

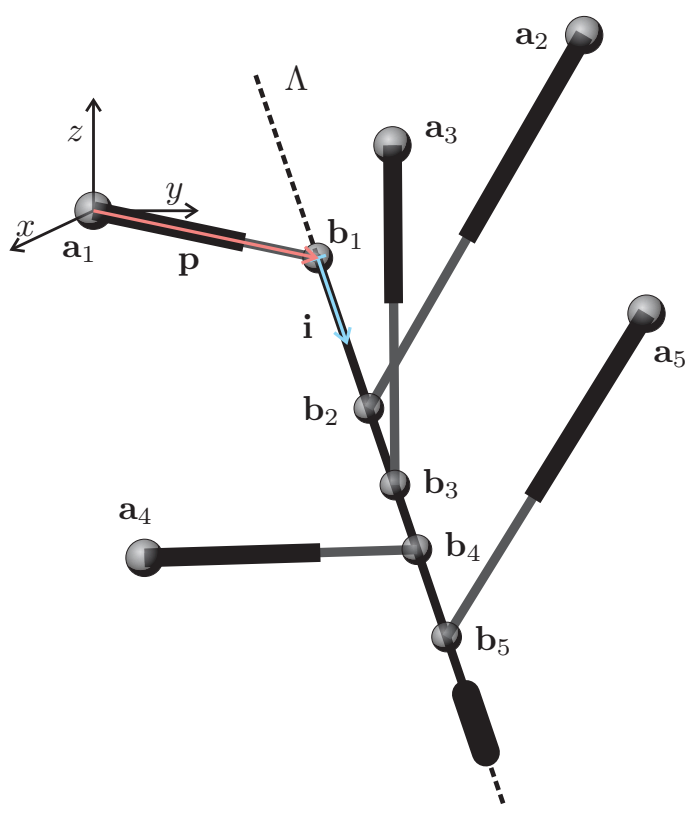

Fig. 2. Notation associated with a pentapod.

rows are the Plücker coordinates of the leg lines [6]:

$$
\mathbf{J}=\left(\begin{array}{c}
\mathbf{c}_{1}^{T} \\
\vdots \\
\mathbf{c}_{6}^{T}
\end{array}\right) \text { where } \mathbf{c}_{i}=\left(\begin{array}{c}
\mathbf{b}_{i}-\mathbf{a}_{i} \\
\mathbf{a}_{i} \times\left(\mathbf{b}_{i}-\mathbf{a}_{i}\right)
\end{array}\right) .
$$

Then, substituting the point coordinates in terms of the introduced notation,

$$
\mathbf{c}_{i}=\left(\begin{array}{c}
p_{x}+r_{i} u-x_{i} \\
p_{y}+r_{i} v-y_{i} \\
p_{z}+r_{i} w-z_{i} \\
z_{i}\left(p_{y}+r_{1} v\right)-y_{i}\left(p_{z}+r_{i} w\right) \\
x_{i}\left(p_{z}+r_{1} w\right)-z_{i}\left(p_{x}+r_{i} u\right) \\
y_{i}\left(p_{x}+r_{1} u\right)-x_{i}\left(p_{y}+r_{i} v\right)
\end{array}\right)
$$

for $i=1, \ldots, 5$.

It is also well-known that when a Stewart platform contains rigid components, the determinant of its Jacobian factors into several terms. For the analyzed Stewart platform, the Jacobian determinant factors as follows:

$$
\operatorname{det}(\mathbf{J})=F_{1}\left(\mathbf{c}_{1}, \ldots \mathbf{c}_{5}\right) F_{2}\left(\mathbf{c}_{6}\right),
$$

where the factor $F_{2}\left(\mathbf{c}_{6}\right)$ depends only on geometric parameters of the sixth leg and platform pose parameters, so the factor $F_{1}\left(\mathbf{c}_{1}, \ldots \mathbf{c}_{5}\right)$ accounts for the singularities of the pentapod embedded in the considered platform. It can be checked, using a computer algebra system, that $F_{1}\left(\mathbf{c}_{1}, \ldots \mathbf{c}_{5}\right)$ - the singularity polynomial of the pentapod - can be expressed as the determinant of the following matrix:

$$
\mathbf{S}=\left(\begin{array}{ccccccc}
1 & u & v & w & p_{x} & p_{y} & p_{z} \\
0 & p_{x} & p_{y} & p_{z} & 0 & 0 & 0 \\
0 & 0 & 0 & 0 & u & v & w \\
r_{2} & x_{2} & y_{2} & z_{2} & r_{2} x_{2} & r_{2} y_{2} & r_{2} z_{2} \\
r_{3} & x_{3} & y_{3} & z_{3} & r_{3} x_{3} & r_{3} y_{3} & r_{3} z_{3} \\
r_{4} & x_{4} & y_{4} & z_{4} & r_{4} x_{4} & r_{4} y_{4} & r_{4} z_{4} \\
r_{5} & x_{5} & y_{5} & z_{5} & r_{5} x_{5} & r_{5} y_{5} & r_{5} z_{5}
\end{array}\right) .
$$


This is a very convenient representation of the singularities of a pentapod because the first three rows depend only on its pose and the remaining four, on the coordinates of the attachments.

Let $\hat{\mathbf{S}}$ denote the $4 \times 7$ matrix formed by the last four rows of $\mathbf{S}$. Since the coefficients of the singularity polynomial of the pentapod are the $4 \times 4$ minors of this matrix, we can conclude this section with the following two observations:

1) If $\hat{\mathbf{S}}$ is rank defective, the pentapod will always be singular irrespective of its leg lengths. In other words, it will be architecturally singular [5].

2) If one of the four rows of $\hat{\mathbf{S}}$ is substituted by another row linearly dependent on these four row vectors, the resulting matrix will have the same $4 \times 4$ minors up to a constant multiple. This observation will allow us, in the next section, to obtain leg substitutions that leave the pentapod singularities invariant.

\section{Singularity-InVariant Leg Substitutions}

We are interested in substituting leg $i$ by another leg with base and platform attachment coordinates $\mathbf{a}=(x, y, z)^{T}$ and $\mathbf{b}=\mathbf{p}^{T}+r \mathbf{i}^{T}$, respectively. Next, we deduce the conditions that $(x, y, z, r)$ must satisfy to leave the singularities of the pentapod unaltered. To this end, consider the matrix

$$
\mathbf{P}=\left(\begin{array}{ccccccc}
r_{2} & x_{2} & y_{2} & z_{2} & r_{2} x_{2} & r_{2} y_{2} & r_{2} z_{2} \\
r_{3} & x_{3} & y_{3} & z_{3} & r_{3} x_{3} & r_{3} y_{3} & r_{3} z_{3} \\
r_{4} & x_{4} & y_{4} & z_{4} & r_{4} x_{4} & r_{4} y_{4} & r_{4} z_{4} \\
r_{5} & x_{5} & y_{5} & z_{5} & r_{5} x_{5} & r_{5} y_{5} & r_{5} z_{5} \\
r & x & y & z & r x & r y & r z
\end{array}\right)
$$

and take $(x, y, z, r)$ such that $\mathbf{P}$ is rank defective. Then, we can substitute any row in $\hat{\mathbf{S}}$ by $(r, x, y, z, r x, r y, r z)$ so that all the $4 \times 4$ minors of the resulting matrix will be equal to those of $\hat{\mathbf{S}}$ up to a constant multiple. Hence, the corresponding singularity polynomial will be also the same, up to a constant factor. In other words, if any of the legs of the analyzed pentapod is substituted by another leg whose attachments coordinates are defined by a set of values for $(x, y, z, r)$ that make $\mathbf{P}$ rank defective, the singularity locus of the pentapod will remain unchanged.

If Gaussian Elimination is applied on $\mathbf{P}^{1}$, the last row of the resulting matrix is:

$$
\frac{1}{P_{567}}\left(\begin{array}{llllllll}
0 & 0 & 0 & 0 & 0 & P_{67} & P_{57} & P_{56}
\end{array}\right),
$$

where $P_{i j}$ is the determinant of the matrix obtained from $\mathbf{P}$ after removing the columns $i$ and $j$, and $P_{i j k}$ the determinant of the matrix formed by the first four rows of $\mathbf{P}$ after removing the columns $i, j$ and $k$. Then, assuming that

\footnotetext{
${ }^{1}$ Gaussian Elimination uses elementary row operations to reduce a given matrix into a rank-equivalent one, with an upper triangular shape. Then, rank deficiency occurs when all the elements of the last row are zero.
}

$P_{567} \neq 0, \mathbf{P}$ is rank defective if, and only if,

$$
\left.\begin{array}{rl}
P_{67} & =\left|\begin{array}{lllll}
r_{2} & x_{2} & y_{2} & z_{2} & r_{2} x_{2} \\
r_{3} & x_{3} & y_{3} & z_{3} & r_{3} x_{3} \\
r_{4} & x_{4} & y_{4} & z_{4} & r_{4} x_{4} \\
r_{5} & x_{5} & y_{5} & z_{5} & r_{5} x_{5} \\
r & x & y & z & r x
\end{array}\right|=0 \\
P_{57} & =\left|\begin{array}{lllll}
r_{2} & x_{2} & y_{2} & z_{2} & r_{2} y_{2} \\
r_{3} & x_{3} & y_{3} & z_{3} & r_{3} y_{3} \\
r_{4} & x_{4} & y_{4} & z_{4} & r_{4} y_{4} \\
r_{5} & x_{5} & y_{5} & z_{5} & r_{5} y_{5} \\
r & x & y & z & r_{y}
\end{array}\right|=0 \\
P_{56} & =\left|\begin{array}{lllll}
r_{2} & x_{2} & y_{2} & z_{2} & r_{2} z_{2} \\
r_{3} & x_{3} & y_{3} & z_{3} & r_{3} z_{3} \\
r_{4} & x_{4} & y_{4} & z_{4} & r_{4} z_{4} \\
r_{5} & x_{5} & y_{5} & z_{5} & r_{5} z_{5} \\
r & x & y & z & r_{z}
\end{array}\right|=0
\end{array}\right\}
$$

Since this system is linear in $x, y$, and $z$, it can be rewritten, after cofactor expansion, in matrix form as:

$$
\left(\begin{array}{ccc}
P_{267}-P_{567} r & -P_{367} & P_{467} \\
P_{257} & -P_{357}-P_{567} r & P_{457} \\
P_{256} & -P_{356} & P_{456}-P_{567} r
\end{array}\right)\left(\begin{array}{l}
x \\
y \\
z
\end{array}\right)=\left(\begin{array}{c}
P_{167} r \\
P_{157} r \\
P_{156} r
\end{array}\right)
$$

whose solution, using Crammer's rule, yields:

$$
\left\{x=\frac{f_{1}(r)}{f(r)}, y=\frac{f_{2}(r)}{f(r)}, z=\frac{f_{3}(r)}{f(r)}\right\},
$$

where $f(r), f_{1}(r), f_{2}(r)$ and $f_{3}(r)$ are cubic polynomials in $r$. Thus, it can be concluded that all singularity-invariant leg substitutions will be defined by a correspondence between points on $\Lambda$ and points on a cubic space curve attached to the base.

\section{Degenerate CAses}

In this section, we analyze the case in which the determinant of the linear system (7) is null, i.e., the case in which the solutions given by (8) are undefined because $f(r)=0$. In this case, two situations arise:

1) System (7) is consistent. One of the equations can be discarded and, for a given value of $r$, infinitely many solutions can be found for $(x, y, z)$ which correspond to points of a line (as they correspond to the intersection of two planes).

2) System (7) is inconsistent. It represents a system of three parallel planes.

If the determinant of the linear system (7) is null, the system is consistent if, and only if, $f_{1}(r)=0, f_{2}(r)=0$, or $f_{3}(r)=0$. Let us suppose that $r_{0}$ is a real root of $f(r)=0$ that makes the system consistent. Then, if one of the platform attachments of the new leg is placed at $\mathbf{b}_{0}=\mathbf{p}+r_{0} \mathbf{i}$, the corresponding base attachment can be placed at any point on the corresponding line in the base. This situation is interesting to build reconfigurable robots where the attachments can be rearranged on actuated guides following the singularityinvariant lines. This allows the manipulator to reconfigure 
itself for different tasks, increasing its useful workspace and maintaining the singularities always at the same location.

TABLE I

THE 4 POSSIBLE ARCHITECTURES FOR A PENTAPOD

\begin{tabular}{|c|c|}
\hline $\begin{array}{c}\text { Number of consistent } \\
\text { real roots }\end{array}$ & Base attachment locus \\
\hline 0 & 1 cubic curve \\
\hline 1 & 1 line and 1 plane conic \\
\hline 2 & 3 non-concurrent lines \\
\hline 3 & 3 concurrent lines \\
\hline
\end{tabular}

We can classify the possible architectures of a pentapod depending on the number of real roots of $f(r)=0$ that lead to a consistent linear system. Depending on this number, the cubic curve obtained for the general case degenerates into a plane conic curve and a line, or a set of lines. Table I summarizes the different possibilities.

\section{EXAMPLES}

\section{A. Generic Case: No Consistent Real Roots}

TABLE II

CoORdinates of THE ATTACHMENTS, $\mathbf{a}_{i}=\left(x_{i}, y_{i}, z_{i}\right)$ AND

$\mathbf{b}_{i}=\mathbf{p}+r_{i} \mathbf{i}$, FOR A PENTAPOD WITH GENERIC ARCHITECTURE

\begin{tabular}{|c|c|c|c|c|}
\hline$i$ & $x_{i}$ & $y_{i}$ & $z_{i}$ & $r_{i}$ \\
\hline \hline 1 & 0 & 0 & 0 & 0 \\
\hline 2 & 6 & 0 & 10 & 1 \\
\hline 3 & 13 & 10 & 12 & 3 \\
\hline 4 & 9 & 16 & 7 & 5 \\
\hline 5 & -3 & 16 & 3 & 7 \\
\hline
\end{tabular}

Consider the pentapod with the attachment coordinates in table II. Substituting them in (7), we get

$$
\left(\begin{array}{ccc}
288 r-6612 & 6306 & 5676 \\
-3136 & 288 r+3904 & 3520 \\
1076 & -2306 & 288 r-1484
\end{array}\right)\left(\begin{array}{l}
x \\
y \\
z
\end{array}\right)=\left(\begin{array}{c}
18816 r \\
16384 r \\
-5504 r
\end{array}\right)
$$

whose determinant is $2654208\left(9 r^{3}-131 r^{2}-r-1365\right)$. The roots are $15.22,-0.33+3.14 i$, and $-0.33-3.14 i$. The evaluation of system (9) for $r=15.22$ yields an inconsistent linear system. As a consequence, the base attachment locus for a leg substituting any of the legs of the analyzed pentapod that would leave its singularity locus invariant is a cubic.

Solving (9) using Crammer's rule gives

$$
\begin{aligned}
& x=\frac{12 r\left(49 r^{2}-240 r-553\right)}{9 r^{3}-131 r^{2}-r-1365}, \\
& y=\frac{256 r\left(-23 r+2 r^{2}+21\right)}{9 r^{3}-131 r^{2}-r-1365}, \\
& z=\frac{-4 r\left(-880 r+4557+43 r^{2}\right)}{9 r^{3}-131 r^{2}-r-1365} .
\end{aligned}
$$

Fig. 3 shows the manipulator and the cubic curve defined by these equations. All legs in gray satisfy the correspondence between $r$ and $(x, y, z)$ through the above curve parameterization, so any of the original pentapod legs can be substituted by any of these legs in gray without modifying the singularity locus of the analyzed pentapod.

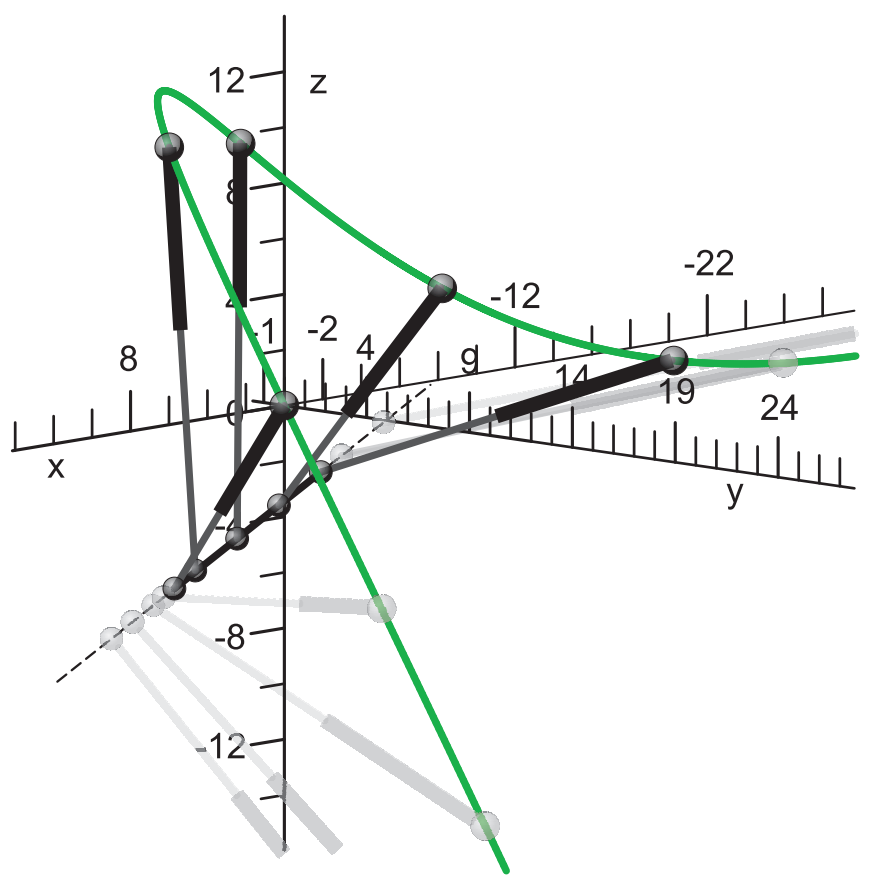

Fig. 3. In general, a singularity-invariant leg substitution is defined by a 1-1 correspondence between the points on the moving platform and the points on a cubic attached to the base. Some candidates for a leg substitution appear in gray.

\section{B. Degenerate Case: Three Consistent Real Roots}

TABLE III

COORDinATES OF THE ATTACHMENTS, $\mathbf{a}_{i}=\left(x_{i}, y_{i}, z_{i}\right)$ AND $\mathbf{b}_{i}=\mathbf{p}+r_{i} \mathbf{i}$, FOR A PENTAPOD WITH DEGENERATE ARCHITECTURE

\begin{tabular}{|c|c|c|c|c|}
\hline$i$ & $x_{i}$ & $y_{i}$ & $z_{i}$ & $r_{i}$ \\
\hline \hline 1 & 0 & 0 & 0 & 0 \\
\hline 2 & 0 & 0 & 0 & 2 \\
\hline 3 & 2 & 2 & -2 & 4 \\
\hline 4 & 4 & -4 & -4 & 5 \\
\hline 5 & 0 & -4 & -4 & 6 \\
\hline
\end{tabular}

Consider the pentapod with the attachment coordinates in Table III. Substituting them in (7), we get

$$
\left(\begin{array}{ccc}
2304-512 r & -128 & 128 \\
-256 & 2688-512 r & 384 \\
256 & 384 & 2688-512 r
\end{array}\right)\left(\begin{array}{l}
x \\
y \\
z
\end{array}\right)=\mathbf{0}
$$

whose determinant is $-134217728(r-5)(r-6)(r-4)$. All the roots are real and make the system consistent. This system has the trivial solution $x=y=z=0$ for any value of $r$. In other words, any leg can be substituted, without modifying the singularities of the analyzed pentapod, by any other with attachments located at $(0,0,0)$ in the base and anywhere in the moving platform.

Now, consider one of the above roots, for example $r=4$. 


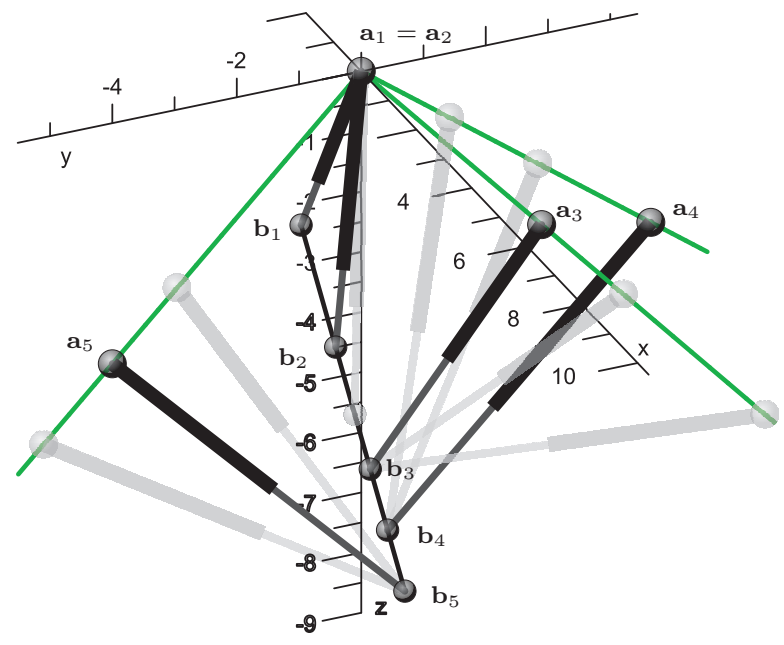

(a)

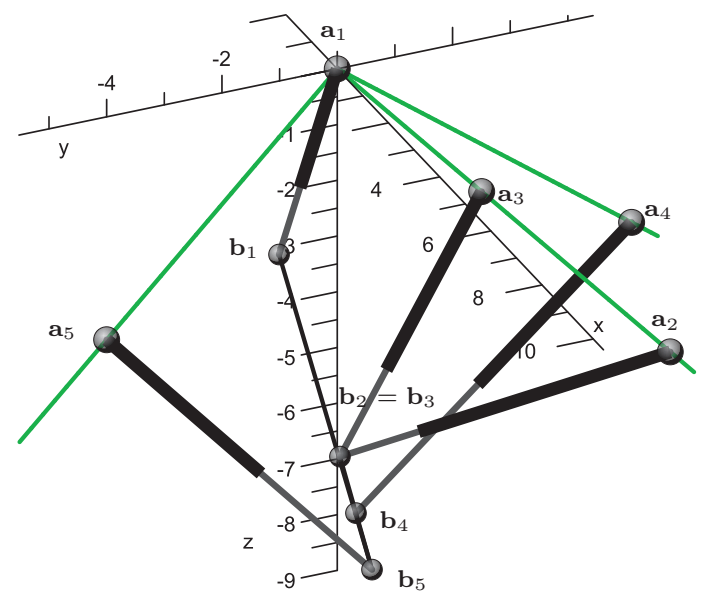

(b)

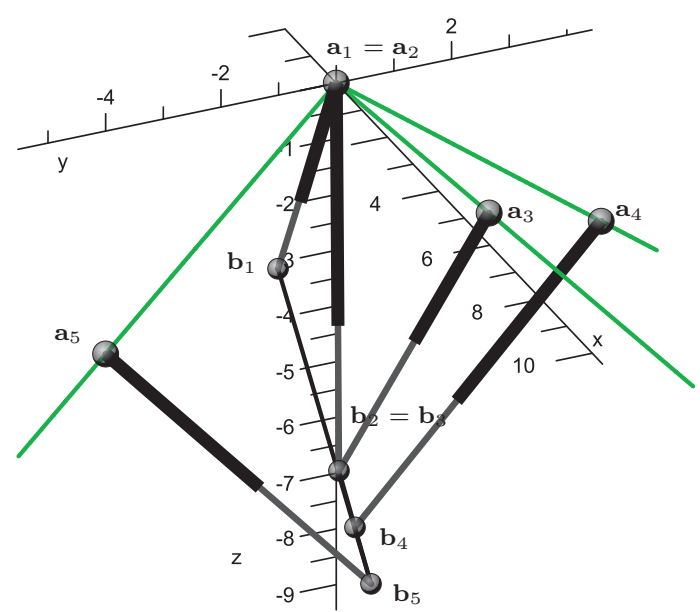

(c)

Fig. 4. Example of a pentapod with degenerate architecture. In this case the cubic curve degenerates into three lines. The base attachments can be moved along these lines without modifying the singularity locus of the pentapod (a). This permits coalescing two attachments in the moving platform (b), or two attachments in the base and the platform at the same time (c).
The substitution of this value in (11) yields

$$
\left.\begin{array}{l}
2 x-y+z=0 \\
2 x-5 y-3 z=0 \\
2 x+3 y+5 z=0
\end{array}\right\}
$$

which is a consistent linear system. That is, the three plane equations intersect at the same line. Solving this system for $x$ and $y$ leads to a parametrization of such line: $\{(x, y, z) \mid$ $x=-t, y=-t, z=t, t \in \mathbb{R}\}$. Proceeding in a similar way for the other two roots, we obtain two more line parameterizations. Summarizing, we have four solution sets, namely:

$$
\begin{aligned}
& \mathrm{s} 1=\{(x, y, z, r) \mid r=4, x=-t, y=-t, z=t, t \in \mathbb{R}\} \\
& \mathrm{s} 2=\{(x, y, z, r) \mid r=5, x=t, y=-t, z=t, t \in \mathbb{R}\} \\
& \mathrm{s} 3=\{(x, y, z, r) \mid r=6, x=0, y=t, z=t, t \in \mathbb{R}\} \\
& \mathrm{s} 4=\{(x, y, z, r) \mid r=t, x=0, y=0, z=0, t \in \mathbb{R}\}
\end{aligned}
$$

In Fig. 4-(a), the legs in gray have attachments whose coordinates are in one of the above solution sets. The legs with platform attachments coordinates $\mathbf{b}_{3}, \mathbf{b}_{4}$ and $\mathbf{b}_{5}$ correspond to solutions in $s_{1}, s_{2}$ and $s_{3}$, respectively. The solution set $s_{4}$ corresponds to legs with base attachment at $(0,0,0)$ and platform attachment anywhere in the moving platform line.

Several equivalent manipulators, from the point of view of their singularities, can be obtained by substituting only leg 2 . For example, in Fig. 4-(b), this leg has been substituted by a leg whose attachments coordinates are in $s_{1}$. Its attachments are $\mathbf{a}_{2}=(4,4,-4)$ and $\mathbf{b}_{2}=\mathbf{p}+4 \mathbf{i}$, thus making coincident $\mathbf{b}_{2}$ and $\mathbf{b}_{3}$. In Fig. 4-(c), leg 2 has been substituted by a leg whose attachments coordinates are in $s_{4}$. In this case, the new attachments have coordinates $\mathbf{a}_{2}=(0,0,0)$ and $\mathbf{b}_{2}=\mathbf{p}+4 \mathbf{i}$ thus making coincident $\mathbf{a}_{1}$ with $\mathbf{a}_{2}$, and $\mathbf{b}_{2}$ with $\mathbf{b}_{3}$.

If actuated guides are placed on the lines defined by s1, s2, and s3, the manipulator can reconfigure its base attachments following singularity-invariant leg rearrangements. This increases its usable workspace because, though singularities remain unchanged, its stiffness does change at each reconfiguration, so that it can be optimized for each specific task at different regions of the workspace.

\section{Degenerate Case: One Consistent Real Root}

TABLE IV

COORdinAtes OF THE ATTACHMENTS, $\mathbf{a}_{i}=\left(x_{i}, y_{i}, z_{i}\right)$ AND $\left.\mathbf{b}_{i}=\mathbf{p}+r_{i} \mathbf{i}\right)$, FOR A PENTAPOD WITH DEGENERATE ARCHITECTURE

\begin{tabular}{|c|c|c|c|c|}
\hline$i$ & $x_{i}$ & $y_{i}$ & $z_{i}$ & $r_{i}$ \\
\hline \hline 1 & 0 & 0 & 0 & 0 \\
\hline 2 & -2 & $2 \sqrt{3}$ & -1 & 1 \\
\hline 3 & -9 & $4 \sqrt{3}$ & -3 & 3 \\
\hline 4 & -8 & 0 & -1 & 5 \\
\hline 5 & -6 & $-2 \sqrt{3}$ & 0 & 7 \\
\hline
\end{tabular}

Consider the pentapod with the attachment coordinates in Table IV. Substituting them in (7), we get 


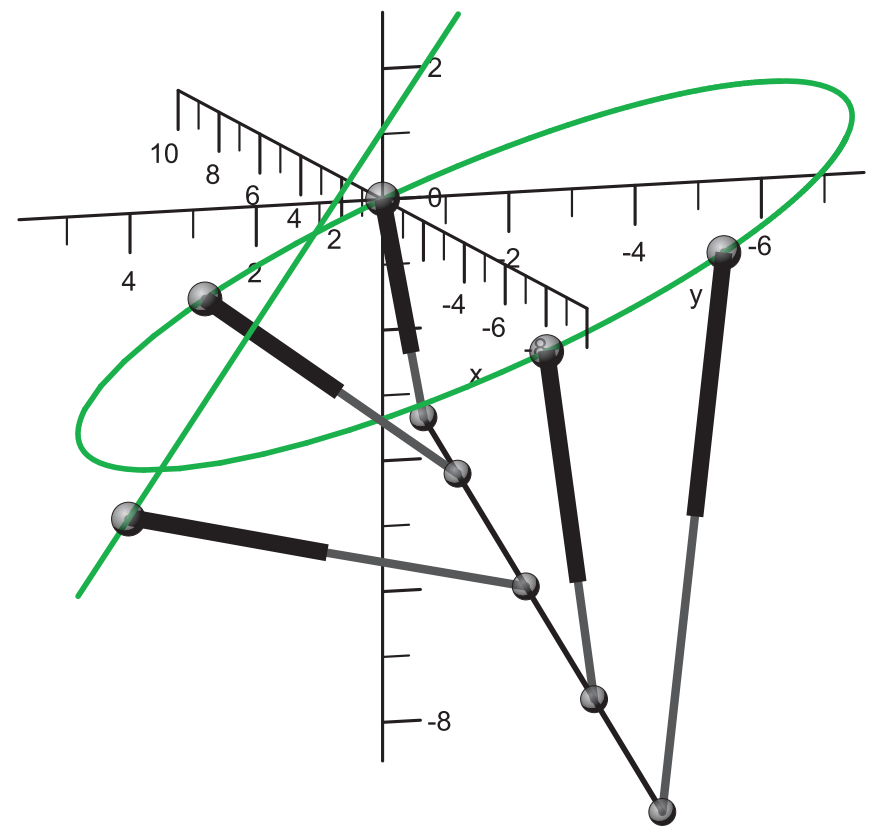

Fig. 5. One consistent real root results in a base attachment locus formed by a line and a conic.

$$
\left(\begin{array}{ccc}
\sqrt{3}(-18 r+70) & 84 & 40 \sqrt{3} \\
-144 & 18 \sqrt{3}(1-r) & 72 \\
20 \sqrt{3} & 24 & \sqrt{3}(50-18 r)
\end{array}\right)\left(\begin{array}{l}
x \\
y \\
z
\end{array}\right)=\left(\begin{array}{c}
24 r \sqrt{3} \\
216 r \\
-24 r \sqrt{3}
\end{array}\right)
$$

The solution of the above system, obtained using Crammer's rule, is

$$
\begin{aligned}
& x=\frac{-4 r(r+11)}{3 r^{2}-14 r+35}, \\
& y=\frac{-12 r(r-5) \sqrt{3}}{3 r^{2}-14 r+35}, \\
& z=\frac{4 r(r-7)}{3 r^{2}-14 r+35} .
\end{aligned}
$$

which corresponds to a conic parameterized in $r$.

The determinant of system (12) is $-5832 \sqrt{3}\left(3 r^{2}-14 r+\right.$ $35)(r-3)$. The only real root is $r=3$. After evaluating (12) for this real root, a consistent degenerate system is obtained whose solution is:

$$
\left\{r=3, x=t-6, y=-\frac{2(t-3) \sqrt{3}}{3}, z=t\right\},
$$

which corresponds to a line parameterized in $t$.

In summary, the locus of the base attachments consists of a line and a conic (see Fig. 5).

\section{CONCLUSIONS}

In this paper, we have introduced a new representation of the singularity polynomial of a pentapod. This new expression reveals to be very convenient because, among other properties, it allows to easily define leg substitutions that keep singularities invariant.
Although finding singularity-invariant leg substitutions does not solve the problem of characterizing the singularities themselves, it provides a lot of insight into its geometric nature. In this context, the obtained leg substitutions are of practical interest for two main reasons:

1) If the singularity locus of the pentapod at hand has already been characterized, it could be interesting to modify the location of its legs to optimize some other characteristics without altering such locus.

2) If the singularity locus of the analyzed pentapod has not been characterized yet, it could be of interest to simplify its geometry by changing the leg attachment locations, as it has been shown in the example presented in Section V-B, thus easing the task of obtaining this characterization.

We have also shown that for any pentapod there is an intrinsic correspondence between the points on the moving platform line and the points on a cubic curve placed on the fixed base. This characterization permits to study degenerate cases that present interesting practical applications.

\section{REFERENCES}

[1] G. F. Bär and G. Weiß, "Kinematic analysis of a pentapod robot," Journal for Geometry and Graphics, Vol. 10, No. 2, pp. 173-182, 2006.

[2] J. Borràs and F. Thomas, "Kinematics of line-plane subassemblies in Stewart platforms," Proc. of the 2009 IEEE Int. Conf. on Robotics and Automation, pp. 4094-4099, 2009.

[3] M.L. Husty and A. Karger, "Self-Motions of Griffis-Duffy Type Parallel Manipulators," Proc. IEEE Intl. Conf. on Robotics and Automation, pp. $7-12,2000$.

[4] X. Kong and C.M. Gosselin, "Generation and Forward Displacement Analysis of Two New Classes of Analytic 6-SPS Parallel Manipulators," Journal of Robotic Systems, Vol. 18, No. 6, pp. 295-304, 2001.

[5] O. Ma and J. Angeles, "Architecture singularities of platform manipulators," Proc. IEEE Intl. Conf. on Robotics and Automation, vol. 2, pp. 1542-1547, 1991.

[6] J.-P. Merlet, "Singular configurations of parallel manipulators and grassmann geometry," International Journal of Robotics Research, vol. 8, no. 5, pp. 45-56, 1989.

[7] J-P. Merlet, Parallel Robots, Springer, 2000.

[8] R. Neugebauer, M. Schwaar, St. Ihlenfeldt, G. Pritschow, C. Eppler, and T. Garber, "New approaches to machine structures to overcome the limits of classical parallel structures," CIRP Annals - Manufacturing Technology, Vol. 51, No. 1, pp. 293-296, 2002.

[9] M. Wecka and D. Staimera, "Parallel kinematic machine tools. Current state and future potentials," CIRP Annals - Manufacturing Technology, Vol. 51, No. 2, pp. 671-683, 2002.

[10] G. Weiß and G.F. Bär, "Singularity investigation of a 5-leg milling robot," EuCoMeS, 1st European Conference on Mechanism Science, Obergurgl, Austria, 2006.

[11] C. Zhang and S.M. Song, "Forward kinematics of a class of parallel (Stewart) platforms with closed-form solutions," Proc. IEEE Intl. Conf. on Robotics and Automation, pp. 2676-2681, 1991. 